\title{
Control of Inter-Dot Electrostatic Coupling by a Side Gate in a Silicon Double Quantum Dot Operating at $4.5 \mathrm{~K}$
}

\author{
Gento Yamahata ${ }^{1 *}$, Tetsuo Kodera ${ }^{1}$, Hiroshi Mizuta ${ }^{2,3,4}$, Ken Uchida ${ }^{3,4}$, and Shunri Oda ${ }^{1,4}$ \\ ${ }^{1}$ Quantum Nanoelectronics Research Center, Tokyo Institute of Technology, 2-12-1 O-okayama, Meguro, Tokyo 152-8552, Japan \\ ${ }^{2}$ School of Electronics and Computer Science, University of Southampton, Highfield, Southampton SO17 1BJ, U.K. \\ ${ }^{3}$ Department of Physical Electronics, Tokyo Institute of Technology, 2-12-1 O-okayama, Meguro, Tokyo 152-8552, Japan \\ ${ }^{4}$ SORST-JST, Chiyoda, Tokyo 102-0075, Japan
}

Received June 24, 2009; accepted July 28, 2009; published online August 21, 2009

\begin{abstract}
We report on electron transport measurements of a lithographically-defined silicon double quantum dot (DQD) coupled in series with a top gate and side gates. The structure of the top gate coupled uniformly to the DQD is suitable for realizing a few-electron regime. The obtained small DQD enables us to observe a clear honeycomb-like charge stability diagram at a temperature of $4.5 \mathrm{~K}$. The validity of the DQD structure is confirmed by theoretical calculations. Furthermore, we demonstrate successful modulation of the inter-dot electrostatic coupling by the side gate. Externally tunable coupling is essential for practical implementation of spin-based quantum information devices.

(C) 2009 The Japan Society of Applied Physics
\end{abstract}

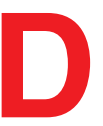

ouble quantum dots (DQDs) are key structures to prepare, manipulate, and detect electron spins toward spin-based quantum information processing. ${ }^{1)}$ Coherent manipulation of individual and coupled electron spin states has been extensively studied in GaAsbased DQD devices. ${ }^{2-5)}$ However, nuclear spins of the host materials cause electron spin decoherence via strong hyperfine coupling. ${ }^{2,3,6)}$ In order to eliminate the effect, group IV materials, such as carbon, silicon $(\mathrm{Si})$ and silicongermanium ( $\mathrm{SiGe}$ ), have recently attracted much attention $^{7-16)}$ because the host materials mainly comprise isotopes which have no nuclear spins. Si systems, particularly, have an advantage in future integration because of the compatibility with conventional $\mathrm{Si}$ metal-oxide-semiconductor (MOS) processes.

One of important prerequisites for investigating electron spin dynamics is to realize few-electron quantum dots (QDs), ${ }^{17)}$ which can be achieved by a formation of small QDs with low electron concentration. Although a fewelectron regime is not achievable in heavily-doped $\mathrm{Si}$ DQDs ${ }^{18,19)}$ one can control electron concentration in $\mathrm{Si}$ DQDs using inversion carriers induced by a top gate. However, since reported Si DQDs using such a top gate structure were defined by two-layer top gates and the upper top gate was not coupled to the Si nanowire with uniformity, ${ }^{11,12}$ ) the inversion carrier concentration was not uniform in the DQD regions. As a result, low electron concentration was difficult to be realized. Furthermore, the signature of the DQD have been observed at a temperature of around $100 \mathrm{mK}$, because of relatively large DQDs formed in the system.

In this paper, we propose a lithographically-defined $\mathrm{Si}$ DQD device with a top gate and side gates and report on clear signatures of electron transport through the Si DQD coupled in series at a temperature of $4.5 \mathrm{~K}$. Since the top gate is coupled uniformly to the Si nanowire, induced electron concentration are controlled to be low. By tuning the voltages applied to the top gate and the side gates, the electron states in the DQD were controlled, leading to the clear observation of the honeycomb-like charge stability diagram. ${ }^{20)}$ The theoretical calculation of the gate capacitances strongly supports the sizes of the DQD. The large

*E-mail address: ygent@neo.pe.titech.ac.jp
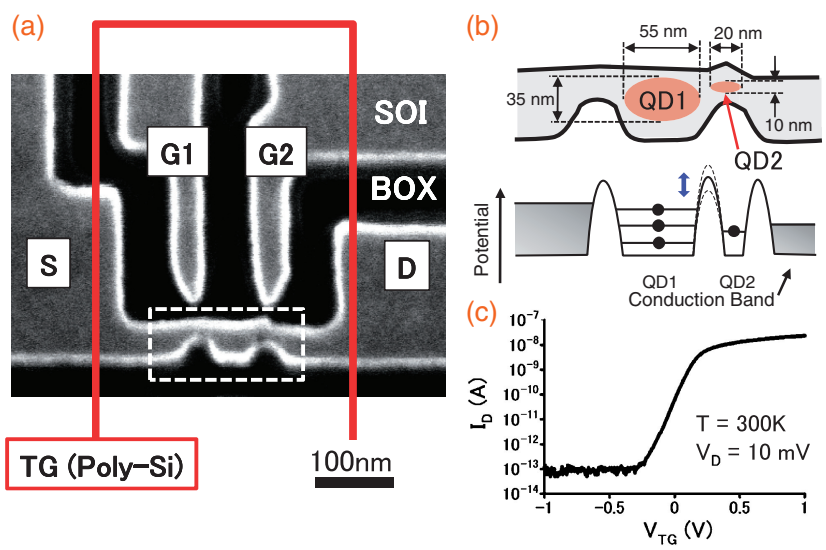

Fig. 1. (a) Scanning electron microscope image of the device before the top gate is formed. (b) Schematic image of the formed quantum dots with the potential diagram. (c) Drain current $I_{D}$ as a function of $V_{\mathrm{TG}}$, where $V_{\mathrm{D}}=10 \mathrm{mV}(T=300 \mathrm{~K})$.

charging energies of the two QDs estimated from the experimental results are attributed to the formation of the small QDs. Furthermore, we demonstrate the modulations of the electrostatic coupling between the two QDs with the voltages applied to the side gate adjacent to the central potential barrier for the first time.

Figure 1(a) shows the scanning microscope image of the device before the formation of the top gate (TG) which is schematically drawn by the red square. The two constrictions in the Si nanowire between the source (S) and the drain (D), indicated by the white broken square shown in Fig. 1(a), and the two side gates (G1, G2) were patterned by electron beam lithography on a 60 -nm-thick Si-on-insulator (SOI) layer, where the thickness of a buried oxide (BOX) is $200 \mathrm{~nm}$. Electron cyclotron resonance reactive ion etching was used to transfer the resist pattern into the SOI, followed by the formation of the gate oxide via thermal oxidation for $30 \mathrm{~min}$ at $1000^{\circ} \mathrm{C}$. The polycrystalline silicon (poly-Si) TG formed by low-pressure chemical vapor deposition was used as an ion implantation mask during the formation of the n-type source and drain regions.

In order to modulate the potential barriers of the device efficiently by the side gates, we designed the extremely 


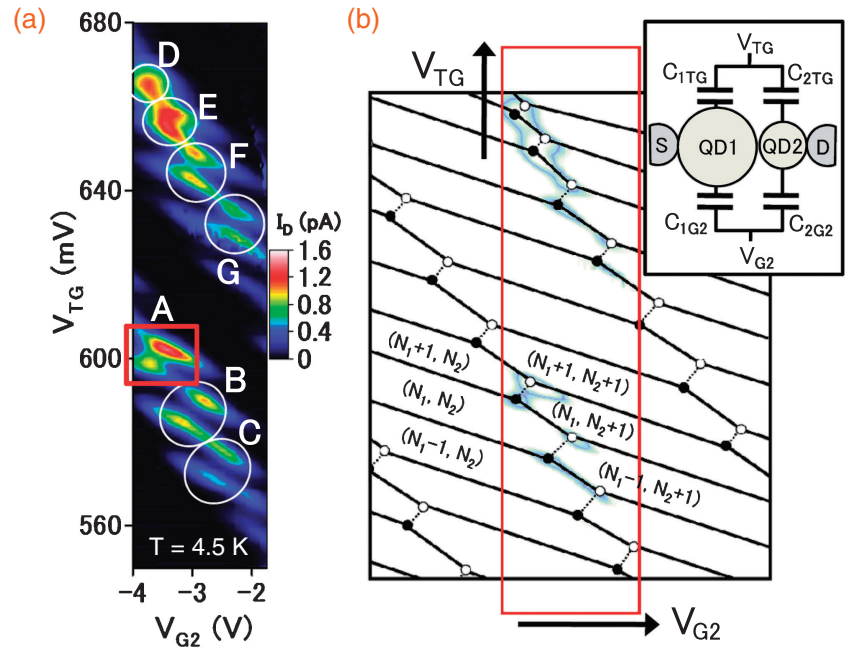

Fig. 2. (a) Contour plots of the drain current $I_{\mathrm{D}}$ as a function of $V_{\mathrm{TG}}$ and $V_{\mathrm{G} 2}$, where $V_{\mathrm{D}}=500 \mu \mathrm{V}(T=4.5 \mathrm{~K})$. (b) Schematic honeycomb diagram corresponding to the experimental result. Inset: equivalent circuit of the DQD capacitively coupled to $V_{\mathrm{TG}}$ and $V_{\mathrm{G} 2}$.

narrow gaps between the side gates and the nanowire region. In addition, the gaps must be filled with $\mathrm{Si}$ dioxide $\left(\mathrm{SiO}_{2}\right)$ because the poly-Si intruding into the gaps strongly screens the gate effects. For the condition of the thermal oxidation, the thickness of the gate oxide is about $30 \mathrm{~nm}$. Therefore the narrow gaps of about $20 \mathrm{~nm}$ were patterned before the gate oxidation [see Fig. 1(a)].

Here, we discuss the formation mechanism of QDs in the device. The width of the two constrictions are controlled to be less than $10 \mathrm{~nm}$ after the gate oxidation for the formation of the potential barriers due to the quantum size effect. Therefore a QD (QD1) is expected to be formed between the two constrictions. In addition, the nanowire adjacent to the side gates often have a bumpy sidewall. In order to obtain a DQD, we adopted the device which has a bump in the right constriction, leading to the formation of another small QD (QD2) as justified below. As a result, the asymmetric DQD is formed as schematically shown in Fig. 1(b) with the corresponding potential diagram, where the reasonable dimensions of the DQD are also denoted.

All measurements were performed using the Agilent 4156A parameter analyzer. Figure 1(c) shows the drain current $\left(I_{\mathrm{D}}\right)$ as a function of the voltages applied to the top gate $\left(V_{\mathrm{TG}}\right)$ with a constant voltage of $10 \mathrm{mV}$ applied to the drain $\left(V_{\mathrm{D}}\right)$ at a temperature of $300 \mathrm{~K}$. $I_{\mathrm{D}}$ shows behavior characteristic of a standard Si MOS field-effect transistor with the top gate.

Figure 2(a) shows the contour plots of $I_{\mathrm{D}}$ as a function of the voltages applied to the side gate $\mathrm{G} 2\left(V_{\mathrm{G} 2}\right)$ and $V_{\mathrm{TG}}$ at a temperature of $4.5 \mathrm{~K}$, where $V_{\mathrm{D}}$ is $500 \mu \mathrm{V}$ and the other gates are grounded. Sequential tunneling through DQDs coupled in series is allowed only at charge triple points, ${ }^{20)}$ where the electrochemical potentials of two QDs and leads are aligned. As a finite bias voltage is applied to DQDs, the conductance exhibits triangularly shaped regions (bias triangle). The observed current peaks correspond to the bias triangles with the charge triple points of the DQD because the honeycomb diagram of the DQD was constructed from the triangular shapes and the positions of the current peaks as shown in
Fig. 2(b). The red rectangle in Fig. 2(b), in which the current peaks are shown by faint colors, corresponds to the contour plots region of Fig. 2(a). Black and white circles shown in Fig. 2(b) are the charge triple points in the DQD. Several stable charge numbers are denoted as $\left(N_{1}, N_{2}\right)$, where $N_{1}$ and $N_{2}$ correspond to the number of electrons confined in QD1 and QD2, respectively.

To evaluate the gate couplings from the honeycomb diagram, we constructed an equivalent circuit as shown in the inset to Fig. 2(b). From the intervals between the charge triple points in Fig. 2(a), the gate capacitances $C_{1 \mathrm{TG}}, C_{2 \mathrm{TG}}$, $C_{1 \mathrm{G} 2}$, and $C_{2 \mathrm{G} 2}$ were estimated to be $13.0,2.73,0.045$, and $0.055 \mathrm{aF}$, respectively. ${ }^{18)} C_{2 \mathrm{G} 2}$ is larger than $C_{1 \mathrm{G} 2}$, indicating the strong coupling between the side gate G2 and QD2. In addition, it was confirmed that the side gate G1 was slightly coupled to only QD1 from the similar measurement of the charge stability diagram by using the side gate G1 instead of the side gate G2. Therefore our hypothesis that QD1 and QD2 are located as shown in Fig. 1(b) is justified.

Here, we calculate $C_{1 \mathrm{TG}}$ and $C_{2 \mathrm{TG}}$ to confirm the validity of the estimated sizes of the DQD with a simplified geometry model, ${ }^{11)}$ which assumes a $\mathrm{Si}$ cylinder covered with a thin $\mathrm{SiO}_{2}$ layer. The gate capacitance $C_{\mathrm{G}}$ in this model is given by $C_{\mathrm{G}}=2 \pi \epsilon L / \ln \left[\left(t_{\mathrm{ox}}+d_{\mathrm{si}} / 2\right) /\left(d_{\mathrm{si}} / 2\right)\right]$, where $\epsilon$ is the permittivity of $\mathrm{SiO}_{2} \quad\left(\epsilon=3.45 \times 10^{-13}\right.$ $\mathrm{F} / \mathrm{cm}), L$ is the wire length, $t_{\mathrm{ox}}$ is the thickness of $\mathrm{SiO}_{2}$, and $d_{\mathrm{si}}$ is the diameter of the Si wire. $L$ and $d_{\mathrm{si}}$ of QD1 (2) are expected to be 55 (20) and 35 (10) nm from the assumed DQD configuration [see Fig. 1(b)]. In addition, we determined $t_{\mathrm{ox}}$ of $30 \mathrm{~nm}$ by using the spectroscopic ellipsometry measurement. As a results, $C_{1 \mathrm{TG}}$ and $C_{2 \mathrm{TG}}$ are calculated as 13.0 and $2.26 \mathrm{aF}$, respectively. These calculated capacitances have a good agreement with the experimental ones, which validates the estimated sizes of the DQD. A slight difference between the calculated $C_{2 \mathrm{TG}}$ and the experimental one is attributed to the actual thickness of the SOI in QD2 (more than $10 \mathrm{~nm}$ ).

In order to estimate the energy scales of QD1 and QD2 from the honeycomb diagram, we magnify one charge triple point indicated by the red square in Fig. 2(a) as shown in Fig. 3(a). The bias triangles are outlined with the bold lines in Fig. 3(a), where the electrochemical potentials of the source, the drain, QD1, and QD2 $\left(\mu_{\mathrm{S}}, \mu_{\mathrm{D}}, \mu_{1}\right.$, and $\left.\mu_{2}\right)$ are illustrated at the vertices of the triangle. In the upper right bias triangle evolved from the triple point as indicated by the white circle in Fig. 3(a), the slight expansion of the current peaks to the left side is due to the co-tunneling current. From the dimensions of the bias triangles $\delta V_{\mathrm{G} 2}$ and $\delta V_{\mathrm{TG}}$, the conversion factors from the gate voltages to the energy scales were extracted. ${ }^{20)}$ As a results, the charging energies of QD1 and QD2 were estimated to be 10.1 and $17.2 \mathrm{meV}$, respectively, by converting the honeycomb cell dimensions to energies. Since these charging energies are larger than those of previous reported DQDs, ${ }^{11-13,15,16)}$ which indicates the formation of the small DQD, we successfully obtain the clear bias triangles at the charge triple points and the honeycomb diagram at a relatively high temperature of $4.5 \mathrm{~K}$.

The electrostatic coupling energy $E_{\mathrm{C} m}$ between QD1 and QD2 is estimated from the gate voltage spacing $\Delta V_{\mathrm{G} 2}^{m}$ between the two charge triple points shown in Fig. 3(a). In 

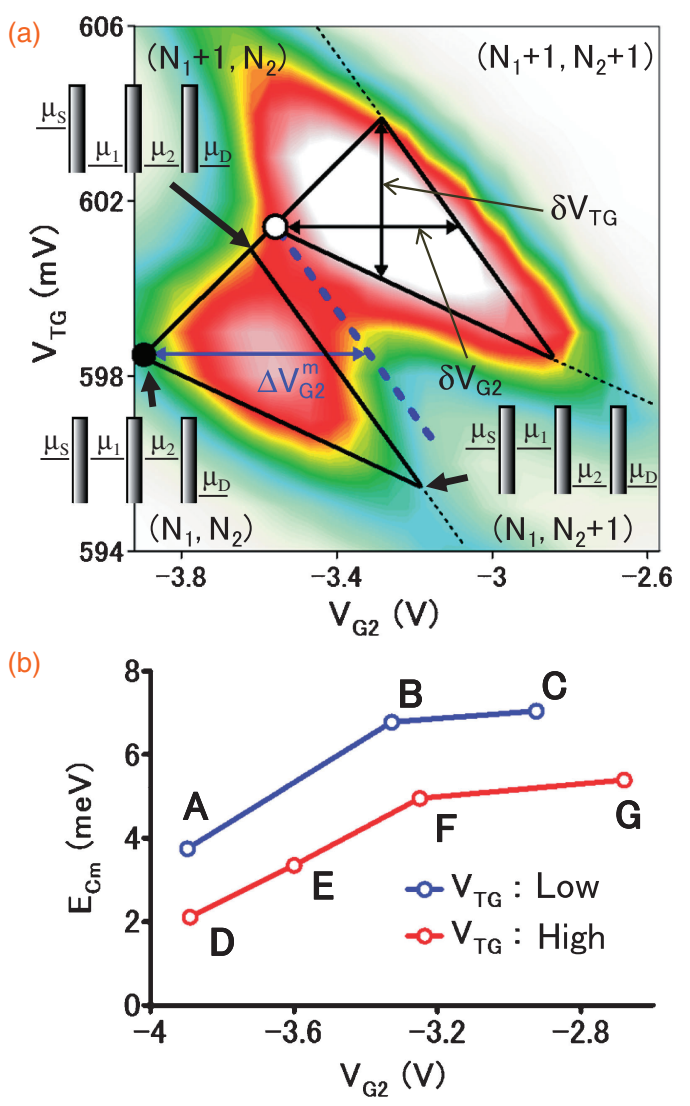

Fig. 3. (a) Bias triangles at the charge triple points corresponding to the red square shown in Fig. 2(a). (b) Electrostatic coupling energies as a function of $V_{\mathrm{G} 2}$ calculated from the distance of the seven charge triple points shown in Fig. 2(a).

the similar way, we extracted $\Delta V_{\mathrm{G} 2}^{m}$ from the 7 charge triple points labeled with $A$ to $G$ with the white circles in the honeycomb diagram shown in Fig. 2(a). Figure 3(b) shows the evaluated $E_{\mathrm{C} m}$ as a function of $V_{\mathrm{G} 2}$, where $E_{\mathrm{C} m}$ decreases with decreasing $V_{\mathrm{G} 2}$. This result demonstrates that the central potential barrier between the two QDs is modulated by the voltages applied to the side gate. The decrease in $E_{\mathrm{C} m}$ results from the decrease in the capacitance of the central barrier, indicating the increase in the thickness of the central barrier at the Fermi energy. As a result, the overlaps of the wavefunctions, namely the tunnel coupling $t$, between QD1 and QD2 are reduced. The modulation of $t$ is directly related to that of the exchange interaction between two electron spins, which is a crucial technique to generate entanglement of spin quantum bits. ${ }^{21-23)}$ The effects of the top gate can be explained by the change of the total capacitances of QD1 and QD2. Since the top gate is coupled to all potential barriers, the total capacitances increase with increasing $V_{\mathrm{TG}}$, resulting in a decrease in $E_{\mathrm{C} m}$.

In conclusion, we have measured the electron transport through the lithographically-defined Si DQD coupled in series with the top gate and the side gates at a temperature of $4.5 \mathrm{~K}$. We observed the clear honeycomb diagram and the charge triple points and identified the formed asymmetric DQD by the theoretical calculations of the gate capacitances. The downscaled DQD is suitable for quantum information devices because a small QD is expected to have large quantum level spacings. In addition, the electrostatic coupling between the DQD is successfully modulated by the voltages applied to the side gate adjacent to the central potential barrier. Externally tunable electrostatic coupling is vital to manipulate and detect the individual spin states in $\mathrm{Si}$ DQDs.

Acknowledgment We thank T. Nagami for helpful discussion, and K. Usami for the device fabrication. This work was partly supported by a Grant-in-Aid for Scientific Research from the Japan Society for the Promotion of of Science (No. 19.10625) and a Grant-in-Aid for Scientific Research from the Ministry of Education, Culture, Sports, Science and Technology, Japan (No. 21710137). G.Y. is supported by JSPS Research Fellowships for Young Scientists.

1) D. Loss and D. P. DiVincenzo: Phys. Rev. A 57 (1998) 120.

2) F. H. L. Koppens, C. Buizert, K. J. Tielrooij, I. T. Vink, K. C. Nowack, T. Meunier, L. P. Kouwenhoven, and L. M. K. Vandersypen: Nature 442 (2006) 766.

3) J. R. Petta, A. C. Johnson, J. M. Taylor, E. A. Laird, A. Yacoby, M. D. Lukin, C. M. Marcus, M. P. Hanson, and A. C. Gossard: Science 309 (2005) 2180.

4) K. C. Nowack, F. H. L. Koppens, Y. V. Nazarov, and L. M. K. Vandersypen: Science 318 (2007) 1430.

5) M. Pioro-Ladriere, T. Obata, Y. Tokura, Y. S. Shin, T. Kubo, K. Yoshida, T. Taniyama, and S. Tarucha: Nat. Phys. 4 (2008) 776.

6) A. V. Khaetskii, D. Loss, and L. Glazman: Phys. Rev. Lett. 88 (2002) 186802.

7) H. O. H. Churchill, F. Kuemmeth, J. W. Harlow, A. J. Bestwick, E. I. Rashba, K. Flensberg, C. H. Stwertka, T. Taychatanapat, S. K. Watson, and C. M. Marcus: Phys. Rev. Lett. 102 (2009) 166802.

8) F. A. Zwanenburg, C. E. W. M. van Rijmenam, Y. Fang, C. M. Lieber, and L. P. Kouwenhoven: Nano Lett. 9 (2009) 1071.

9) N. Shaji, C. B. Simmons, M. Thalakulam, L. J. Klein, H. Qin, H. Luo, D. E. Savage, M. G. Lagally, A. J. Rimberg, R. Joynt, M. Friesen, R. H. Blick, S. N. Coppersmith, and M. A. Eriksson: Nat. Phys. 4 (2008) 540.

10) F. Molitor, S. Dröscher, J. Güttinger, A. Jacobsen, C. Stampfer, T. Ihn, and K. Ensslin: Appl. Phys. Lett. 94 (2009) 222107.

11) A. Fujiwara, H. Inokawa, K. Yamazaki, H. Namatsu, Y. Takahashi, N. M. Zimmerman, and S. B. Martin: Appl. Phys. Lett. 88 (2006) 053121.

12) H. Liu, T. Fujisawa, H. Inokawa, Y. Ono, A. Fujiwara, and Y. Hirayama: Appl. Phys. Lett. 92 (2008) 222104.

13) W. H. Lim, H. Huebl, L. H. W. van Beveren, S. Rubanov, P. G. Spizzirri, S. J. Angus, R. G. Clark, and A. S. Dzurak: Appl. Phys. Lett. 94 (2009) 173502.

14) V. C. Chan, T. M. Buehler, A. J. Ferguson, D. R. McCamey, D. J. Reilly, A. S. Dzurak, R. G. Clark, C. Yang, and D. N. Jamieson: J. Appl. Phys. 100 (2006) 106104.

15) C. B. Simmons, M. Thalakulam, B. M. Rosemeyer, B. J. V. Bael, E. K. Sackmann, D. E. Savage, M. G. Lagally, R. Joynt, M. Friesen, S. N. Coppersmith, and M. A. Eriksson: arXiv:0905.1647.

16) Y. Hu, H. O. H. Churchill, D. J. Reilly, J. Xiang, C. M. Lieber, and C. M. Marcus: Nat. Nanotechnol. 2 (2007) 622.

17) R. Hanson, L. P. Kouwenhoven, J. R. Petta, S. Tarucha, and L. M. K. Vandersypen: Rev. Mod. Phys. 79 (2007) 1217.

18) G. Yamahata, Y. Tsuchiya, S. Oda, Z. A. K. Durrani, and H. Mizuta: Jpn. J. Appl. Phys. 47 (2008) 4820.

19) T. Kodera, T. Ferrus, T. Nakaoka, G. Podd, M. Tanner, D. Williams, and Y. Arakawa: Jpn. J. Appl. Phys. 48 (2009) 06FF15.

20) W. G. van der Wiel, S. D. Franceschi, J. M. Elzerman, T. Fujisawa, S. Tarucha, and L. P. Kouwenhoven: Rev. Mod. Phys. 75 (2002) 1.

21) G. Burkard, D. Loss, and D. P. DiVincenzo: Phys. Rev. B 59 (1999) 2070.

22) T. Hatano, M. Stopa, and S. Tarucha: Science 309 (2005) 268.

23) T. Kodera, K. Ono, Y. Kitamura, Y. Tokura, Y. Arakawa, and S. Tarucha: Phys. Rev. Lett. 102 (2009) 146802. 